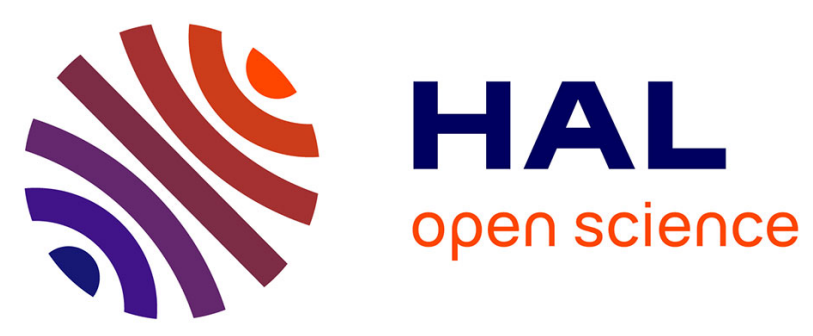

\title{
Damage Investigation in A319 Aluminium Alloy by X-ray Tomography and Digital Volume Correlation during In Situ High-Temperature Fatigue Tests
}

Nora Dahdah, Nathalie Limodin, Ahmed El Bartali, Jean-Francois Witz, Rian Seghir, Eric Charkaluk, Jean-Yves Buffiere

\section{To cite this version:}

Nora Dahdah, Nathalie Limodin, Ahmed El Bartali, Jean-Francois Witz, Rian Seghir, et al.. Damage Investigation in A319 Aluminium Alloy by X-ray Tomography and Digital Volume Correlation during In Situ High-Temperature Fatigue Tests. Strain, 2016, 52 (4), pp.324-335. 10.1111/str.12193 . hal01419328

\section{HAL Id: hal-01419328 \\ https://hal.science/hal-01419328}

Submitted on 23 Oct 2019

HAL is a multi-disciplinary open access archive for the deposit and dissemination of scientific research documents, whether they are published or not. The documents may come from teaching and research institutions in France or abroad, or from public or private research centers.
L'archive ouverte pluridisciplinaire HAL, est destinée au dépôt et à la diffusion de documents scientifiques de niveau recherche, publiés ou non, émanant des établissements d'enseignement et de recherche français ou étrangers, des laboratoires publics ou privés. 


\title{
Damage investigation in a319 aluminium alloy by $X$-ray tomography and digital volume correlation during in situ high temperature fatigue tests
}

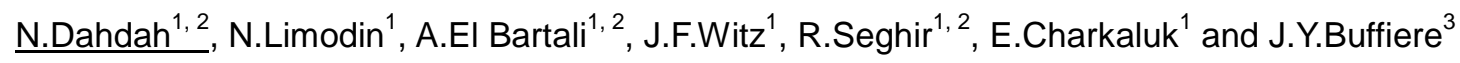 \\ ${ }^{1}$ Laboratoire de Mécanique de Lille (LML), FRE 3723, 59650 Villeneuve d'Ascq, France \\ ${ }^{2}$ Ecole Centrale de Lille, Cité Scientifique, 59650 Villeneuve $d^{\prime}$ Ascq, France \\ ${ }^{3}$ Laboratoire Matériaux, Ingénierie et Sciences (MATEIS), INSA Lyon, CNRS UMR 5510, Avenue Jean Capelle, 69621 \\ Villeurbanne cedex, France
}

\begin{abstract}
The aim of this work is to analyse the strain fields heterogeneity in an aluminium alloy subjected to low cycle fatigue at high temperature. In the cylinder heads produced by a lost foam casting process, the microstructure of the studied alloy consists in hard intermetallic phases and large gas and microshrinkage pores. In order to study the influence of this complex $3 \mathrm{D}$ microstructure on fatigue crack initiation and propagation at $250^{\circ} \mathrm{C}$, an experimental protocol using laboratory and synchrotron tomography, Finite Element simulation and a new Digital Volume Correlation platform has been used. The results showed the role of pores in the crack nucleation and highlighted the importance of hard phases in the crack propagation thanks to the resolution on the DVC measurement.
\end{abstract}

Keywords: Fatigue, A319, Digital Volume Correlation, in situ synchrotron X-ray tomography, High temperature

\section{INTRODUCTION}

Aluminium alloys are extensively used in the automotive industry, especially the Al-Si alloy for different engine parts. Due to its good castability and mechanical properties, the A319 alloy is one of the preferred choice in automotive applications notably for the cylinder head. This part and in particular the inter-valve zone in fire deck, is subjected to severe thermomechanical stresses with temperature gradient from 20 to $250^{\circ} \mathrm{C}$ and high cyclic loadings that can lead to component failure in service [1]. In the automotive industry, the conventional die casting process is progressively being replaced by the Lost Foam Casting (LFC) process for geometry optimization, cost reduction and consumption control purposes. The main benefits of LFC over conventional casting are its ability to produce complex shapes with internal sections at reduced grinding and finishing costs [2]. However, aluminium alloy automotive parts produced by the LFC process have a coarser microstructure and more porosity defects than parts produced with conventional casting processes 
at faster cooling rates. As a result, the A319 cast Aluminium-Silicon alloy produced by LFC process and used for automotive cylinder heads has a multiscale microstructure: porosity with a size that ranges from small gas pores to large microshrinkage cavities, iron intermetallics, eutectic $\mathrm{Al}_{2} \mathrm{Cu}$ and eutectic silicon (Fig.1.a). Above a critical size, pores [3] play a decisive role on the fatigue properties by providing preferential crack initiation sites. However, an influence of oxides [4], iron-based intermetallics [5] and Si particles [6] are also observed. As a matter of fact, cracks can initiate at any large pores or hard phases present in bulk. The coarser microstructure produced by the LFC process has an effect on the mechanical properties and can drastically reduce the fatigue lifetime of the alloy. 2D studies and post-mortem observations (Fig.1.b) reveal that the damage mechanisms are strongly limited to the presence of hard phases [7]. However in this kind of material, the crack often nucleates inside the material; therefore, it is essential to characterize the mechanisms in 3D. In order to study the influence of the LFC microstructure upon the low cycle fatigue behaviour, an experimental protocol has been set up using X-ray tomography and 3D Digital Volume Correlation (DVC).
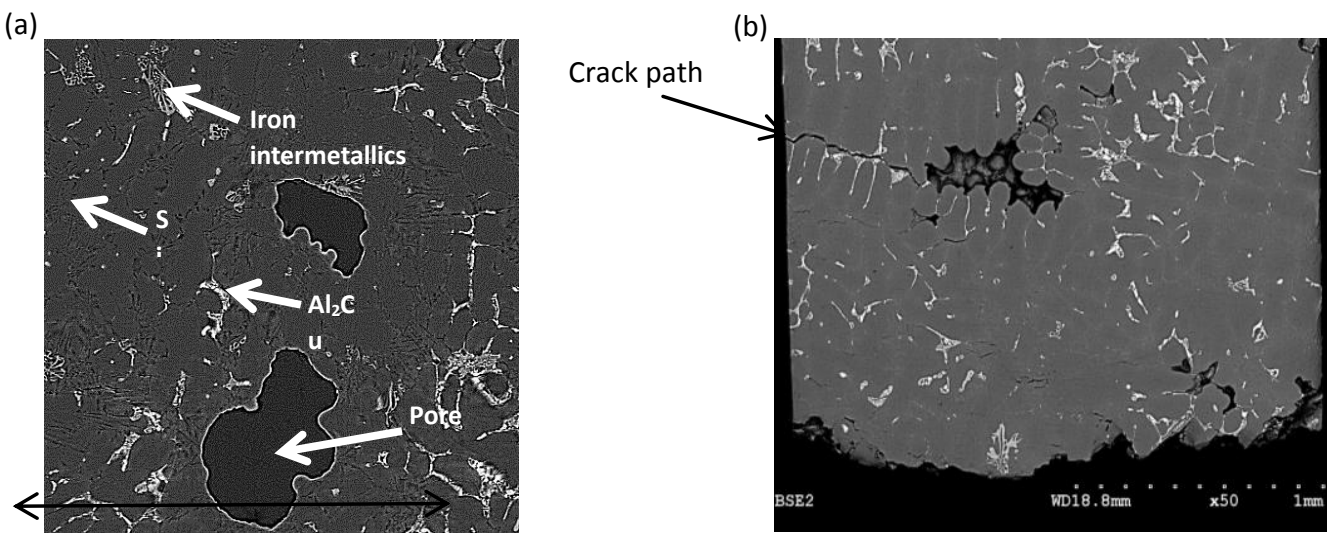

$1.7 \mathrm{~mm}$

Figure 1: (a) Reconstructed tomographic slice (synchrotron tomography) showing the various microstructural features of alloy A319 produced by LFC process, (b) SEM optical microscopy showing crack propagation from an internal pore along hard phases

This paper presents first the experimental protocol used for in-situ fatigue tests at high temperature thanks to Synchrotron tomography and DVC. In a second part, the strain and displacement fields are analysed by comparing the experimental and computed fields under monotonous loading, i.e. under the first half cycle of fatigue, and also by following the evolution of the fatigue crack in the specimen.

\section{EXPERIMENTAL PROCEDURE}

\subsection{X-RAY TOMOGRAPHY}

Small dog bone specimens were cut out from automotive cylinder heads in the inter-valve area (Fig.2.a). A preliminary characterization by laboratory X-ray microtomography was performed at the MATEIS laboratory (Lyon, France) with a 80 $\mathrm{kV}$ acceleration voltage to ensure a $10 \%$ transmission of the X-ray beam through the cross-section of the sample. The scan was made at a medium resolution with a voxel size of $4.5 \mu \mathrm{m}$ and an acquisition time per image of 500 ms; these medium resolution images allow to: (1) select the most suitable specimens, (2) characterize in 3D the population of defects in the 
specimen gauge length, (3) obtain a 3D mesh of the porous aluminium matrix in order to compute the associated strain fields using a finite element software (Abaqus). These preliminary steps help focusing the scanned volumes to a region of interest where cracks are more likely to initiate. Low Cycle Fatigue tests were then performed under synchrotron X-ray tomography at the European Synchrotron Radiation Facility (ID19 beamline) using an in-situ test rig [8] developed at MATEIS laboratory (see Fig.3).

(a)

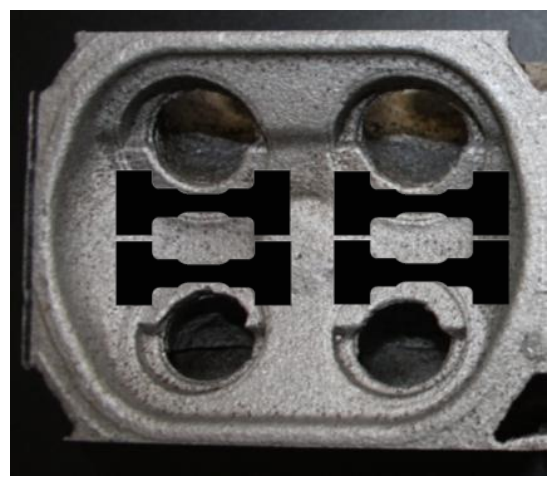

(b)

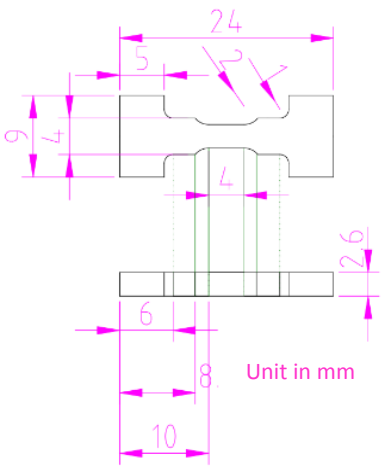

Figure 2: (a) Optical image of the intervalve region of a cylinder head where 36 fatigue samples have been extracted by electro discharge machining; (b) Geometry of the specimen (dimensions in $\mathrm{mm}$ )

Fatigue machine with the furnace

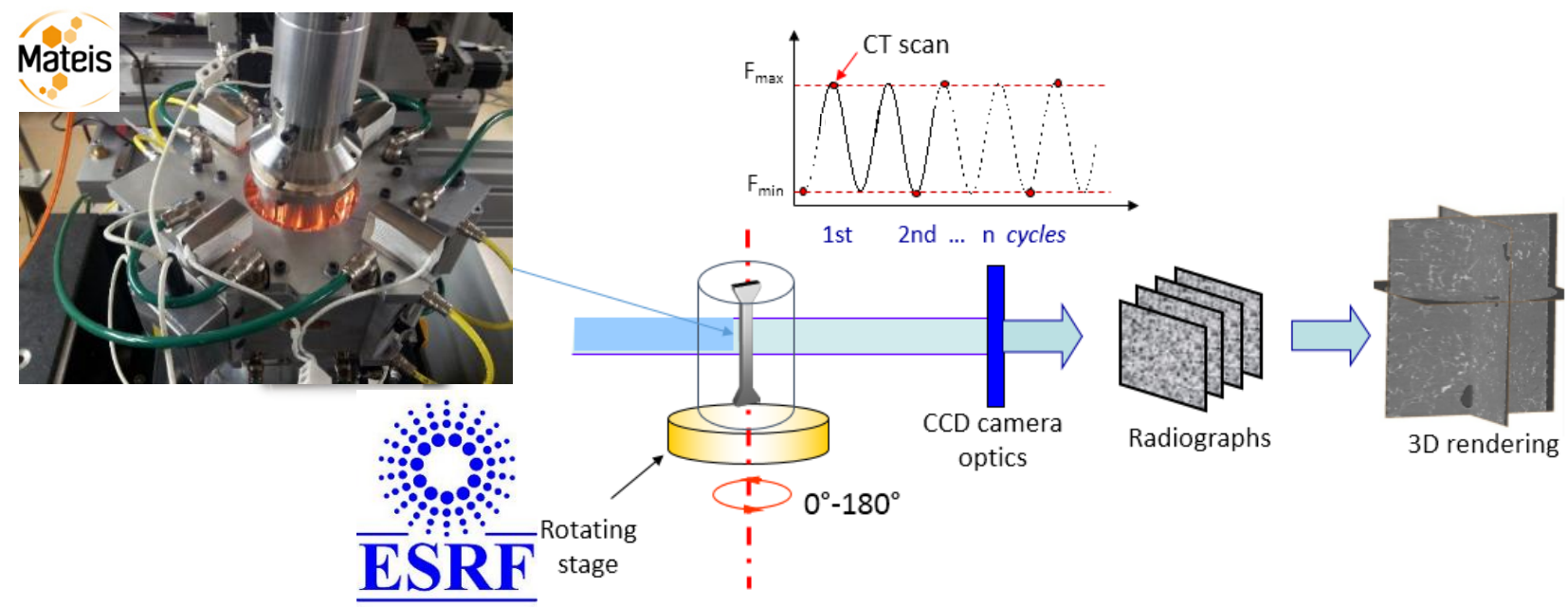

Figure 3: In-situ experimental set-up showing the principle of the tomography

In order to study crack initiation and growth in bulk at high temperatures that are representative of the in-service loadings of cylinder heads, i.e. 150,200 and $250^{\circ} \mathrm{C}$, a new furnace [8] has been realized by the Centre des Matériaux Ecole des Mines de Paris with four halogen lamps [9]. Images were acquired in pink beam mode [10] (E=35 keV) with a CMOS PCO Dimax detector (2048 pixels ${ }^{2}$ ) at $2.75 \mu \mathrm{m}$ voxel size. The scan duration of $45 \mathrm{~s}$ (2000 radiographies) was short enough to avoid creep/relaxation effects at the temperatures investigated $\left(T<250^{\circ} \mathrm{C}\right)$. Seven fatigue specimens with a $2 \times 2 \mathrm{~mm}^{2} \mathrm{cross}$ section (Fig.2.b) were tested: 2 at $150^{\circ} \mathrm{C}, 2$ at $200^{\circ} \mathrm{C}$ and 3 at $250^{\circ} \mathrm{C}$. Uniaxial fatigue tests at constant stress amplitude were performed with a load ratio of 0.1 and a maximum stress of the order of $150 \%$ of the yield stress at the corresponding temperature. Tests were conducted until failure with periodic interruptions in order to detect and follow fatigue crack 
initiation and growth through tomographic scans which were recorded under minimum and maximum load of the considered cycle. All the 3D images were reconstructed by using both the classical filtered backprojection algorithm [11] and the Paganin reconstruction algorithm [12] to have a better contrast between the different phases including eutectic Silicon. For the analysis, only the images reconstructed in Paganin were used. The grey level images were cropped to reduce the amount of data and the time needed to process them. Synchrotron tomography allowed visualizing the eutectic $\mathrm{Al}-\mathrm{Al}_{2} \mathrm{Cu}$, iron based intermetallic phases and above all eutectic Si (see Fig.1.a), which could not be distinguished with laboratory tomography (see Fig.4). Thus, the total volume fraction of hard inclusions that can serve as natural markers for DVC is about $17 \%$ including about $9 \%$ of eutectic Si [30]. In comparison, in a nodular cast iron where the spherical graphite nodules are the only natural markers, the measurement accuracy of DVC is virtually the same either by using laboratory [13] or Synchrotron tomography [14]. In A319 alloy, Synchrotron tomography allows a good contrast to be achieved in the interdendritic space where strain heterogeneity is expected to occur during the fatigue test. As more natural tracers are observed with SR-CT than with lab-CT, a higher measurement accuracy could be obtained for the former with a smaller uncertainty and a higher spatial resolution (ref 3DMS Long). The microstructure of A319 alloy provides a suitable natural speckle for DVC. The 3D displacement field was measured with the DVC technique [15]. In the present study, a 3D image correlation platform called YADICS [16] recently developed at LML laboratory (Lille, France) has been used. This platform is based on $\mathrm{C}_{+}+$and it has been optimized to process large 3D volumes in a limited time. In this section, the finite element simulation is first detailed then the DVC method is explained.
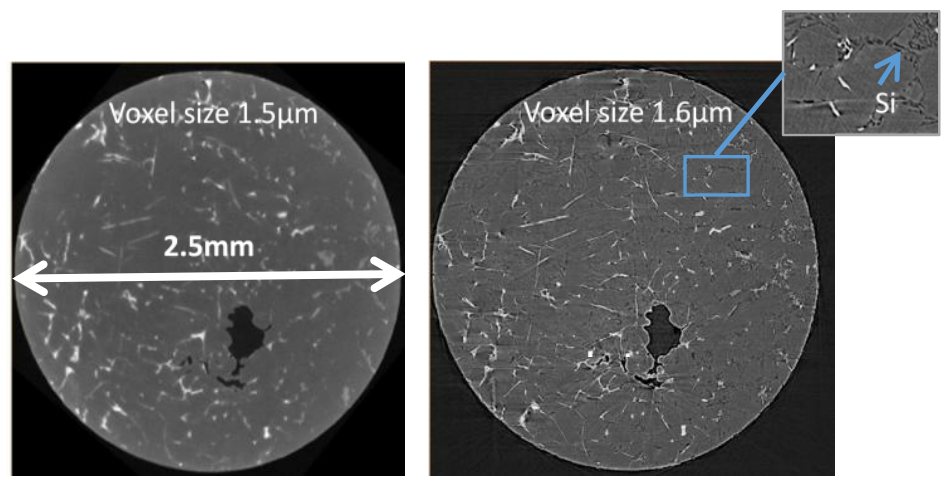

Figure 4: Slice of an A319 alloy from: (a) Laboratory tomography and (b) Synchrotron tomography allowing the characterization of the different phases. The eutectic Si can be distinguished only with Synchrotron tomography.

\subsection{Finite ElEment Simulation}

FEM simulation was performed before the in-situ fatigue test in order to predict the most strained area where crack initiation is expected. 3D images obtained by laboratory tomography were used to generate microstructurally realistic meshes of the microstructure within the pristine samples (pores + matrix, no intermetallic second phase) thanks to Avizo software (Fig.5.a). A triangular surface was reconstructed, the minimum and maximum sizes of the triangle element were set as 0.2 and 10 pixels respectively. The mesh is built in such a way that elements of the mesh generated around the pores have a smaller size than in other areas in order to decrease the number of elements. Then, a volumetric tetrahedral grid was build from the triangular surface mesh (Fig.5.b). Those meshes were used to perform Finite Element (FE) simulation of the strain/stress distribution during monotonic loading (elasto-plastic calculation), i.e. at the maximum load of the first half-cycle (Fig.5.c). In this way, a von 
Mises type elasto-plastic constitutive law has been used, with an isotropic hardening. The entire stress-strain curve coming from a tensile test has been introduced in the Abaqus software. A displacement of $\mathrm{I}=0.018 \mathrm{~mm}$ is applied on upper and lower side of the FE model to obtain a maximum strain $\varepsilon=0.4 \%$ at a length of the specimen $L=9 \mathrm{~mm}$. In order to localize potential critical area, the cumulated plastic strain distribution is computed and plotted, as shown on Figure 5.c. This FE simulation are therefore of great help to define a consistent zone of interest for the tests.

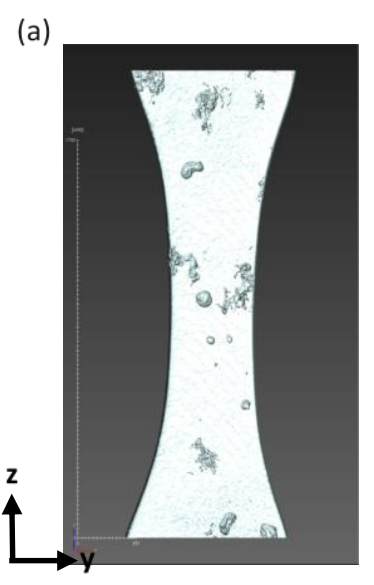

(b)

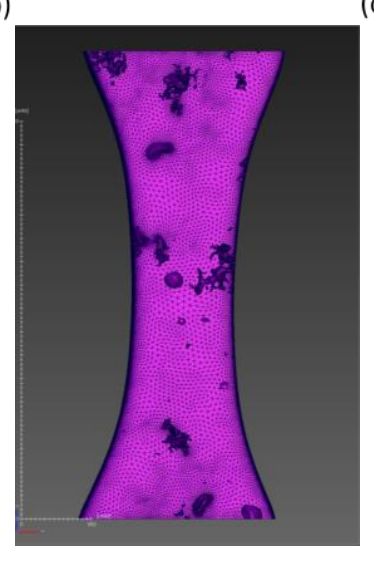

(c)

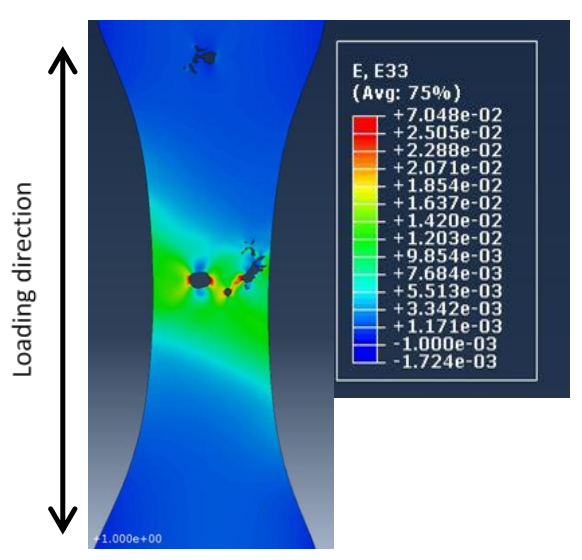

Figure 5 : (a) 3D rendering of pores with Avizo software, (b) 3D volume mesh (5M tetrahedron), (c) FE calculation with Abaqus software showing Ezz

\subsection{Digital Volume Correlation (DVC)}

The DVC technique is a 3D extension of the well-developed DIC method and their principles are similar. DVC was developed from the end of 1990s [17], and is now widely used due to the development of 3D advanced imaging devices [18] [19] [20]. The principle of DVC is to find the transformation between a fixed image and a moving one for a given metric using an optimization scheme (Fig.6). To identify the transformation, several steps are necessary: define a metric, choose a sampling and an interpolator, then an optimizer and finally a regularization method. The first one is to define a metric with the type of the transformation as a variable, the fixed image and the moving image. The DVC formulation used here is based on texture conservation recalled in Equation 1, where $\mathbf{x}$ is the vector denoting the position of any voxel and $\mathbf{u}$ is the unknown displacement vector field. Each image is identified by its grey level function representing the volume of the sample: $f$ for the reference 3D image taken before deformation, $g$ for the deformed 3D image taken after deformation [21] [22]. In this case, we used the optical flow equation which assumes that the grey level variations are due to the displacement of the material points only:

$$
f(\mathbf{x})=g(\mathbf{x}+\mathbf{u}(\mathbf{x}))
$$

In this study, global (rigid and homogeneous) and elastic transformations were chosen in order to compute the metric defined by the Sum of Squared Difference (SSD) (Eq.2) which corresponds to a least squares problem. It consists in finding the best displacement field $\mathbf{u}(\mathbf{x})$ by minimizing:

$$
\operatorname{SSD}(\mathrm{u}, \mathrm{f}, \mathrm{g})=\frac{1}{\Omega} \sum_{\mathbf{x} \in \Omega}(\mathrm{f}(\mathrm{x})-\mathrm{g}(\mathbf{x}+\mathbf{u}(\mathbf{x})))^{2}
$$


With YADICS software, the metric is evaluated on the whole image: it is a total sampling. Then, an optimizer method has to be chosen and in the present case a gradient descent method is used for optimization. The gradient descent method allows to converge quickly to a minima. The first step is to compute the gradient of the metric SSD regarding the transform parameters (Eq.3):

$$
\frac{\partial \operatorname{SSD}(\mathbf{u}, \mathrm{f}, \mathrm{g})}{\partial \mathbf{u}}=\frac{2}{\Omega} \sum_{\mathbf{x} \in \Omega}(\mathrm{f}(\mathrm{x})-\mathrm{g}(\mathbf{x}+\mathbf{u}(\mathbf{x}))) \frac{\partial \mathrm{g}(\mathbf{x}+\mathbf{u}(\mathbf{x}))}{\partial \mathbf{u}}
$$

The optimization can be performed with any iterative numerical algorithm. Once the metric gradient has been computed, an optimization strategy is chosen. The gradient method principle is explained in (Eq.4):

$$
\mathbf{u}_{\mathrm{k}+1}=\mathrm{u}_{\mathrm{k}}+\boldsymbol{\alpha}_{\mathrm{k}} \mathrm{d}_{\mathrm{k}}(\mathrm{Eq} .4)
$$

The gradient step $\boldsymbol{\alpha}_{\mathrm{k}}$ can be constant or updated at every iteration and $\mathbf{d}_{\mathrm{k}}$ defined by:

$$
\mathbf{d}_{\mathrm{k}}=-\gamma_{\mathrm{k}} \frac{\partial \operatorname{SSD}(\mathrm{u}, \mathrm{f}, \mathrm{g})}{\partial \mathbf{u}}
$$

where $\gamma_{\mathrm{k}}=1$ and $S S D$ the metric.

Fixed image
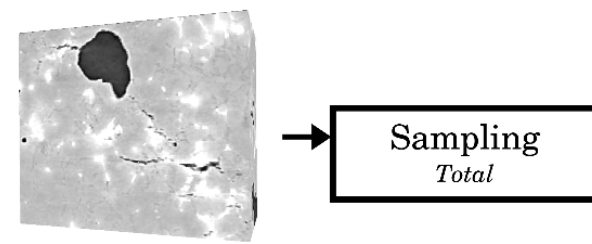

Moving image
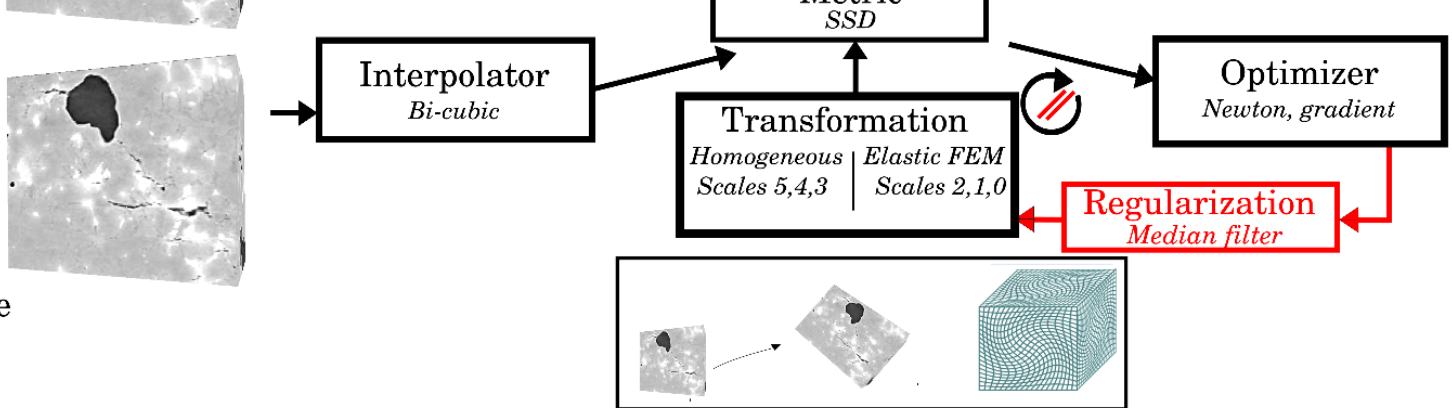

Figure 6 : The basic registration components of YADICS [18] image correlation platform at LML (see the text for details)

One notices that optimization rules used herein is not the classical Gauss-Newton used in [11], [17]. In order to treat such large volumes with a high Degree of Freedom (superior to 1 million), it is easier to use a steepest gradient algorithm [23] as there is no matrix problem to solve [24]. Finally, a bi-cubic interpolator is employed to apply the transformation to the moving image at each iteration. Cachier et al. [25] demonstrated that the problem of minimizing image and mechanical energy can be reformulated in solving the energy image then applying a Gaussian filter at each iteration. Yadics software uses this strategy and adds a median filter (see the arrow in Fig.6 from "Regularization" to "Transformation"), here, with a window of $3 \times 3 \times 3$ elements, allowing to avoid local minima while preserving discontinuities. A median filter removes the noisy pixels by replacing the tested pixel with the median value of the pixels in the window [26]. The median filtering is a 
nonlinear filtering technique that is known for preserving sharp changes in signal and for being particularly effective in removing impulsive noise [27]. One effective use of median filters has been the reduction of high-frequency and impulsive noise in digital images without the edge destruction associated with linear filters. Thus, the discontinuities, i.e. the sharp change of the displacement field, are preserved because it is a no diffusive method [28].

The correlation is based on a multiscale resolution strategy like a pyramid scheme (see Fig.7) allowing to save time as the image contains much fewer voxels in the first resolution levels. In the present case, six resolutions or scales were used; the

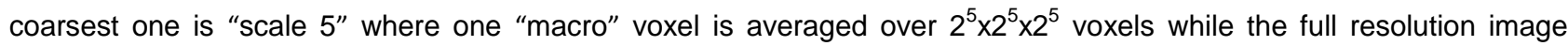
corresponds to "scale 0". The correlation calculation is a sequence of Optical Flow Integrated (OFI) and Finite Element methods (OFFEM). For easy and fast convergence at the coarsest scales, only global transformations are searched for, using OFI algorithms while at the finest scales, the more computing intensive OFFEM algorithm was used to compute local displacements. The correlation sequence per image couple was defined with the following analysis sequence OFI_H, OFI_H, OFI_H, OFFEM4, OFFEM4, OFFEM4 where:

- $\quad$ OFI_H is defined by the OFI method, and assumes a homogeneous deformation such as rigid body motions, i.e, translations (Tx, Ty, Tz) and rotations (Rx,Ry,Rz), and global strains (Exx, Eyy, Ezz, Exy, Exz, Eyz). It measures the similarity by using Sum of Squared Differences (SSD). OFI was used for global transformation for scales 5 to 3 , as it can reduce the dimensionality of the problem and it has a low sensitivity to noise.

- OFFEM is the Optical Flow elastic transformation based on Finite Element Methods, it is used for the elastic deformation, as it has a good spatial coherence. OFFEM4 mean the used element size is $4 \times 4 \times 4$ voxels, respectively.

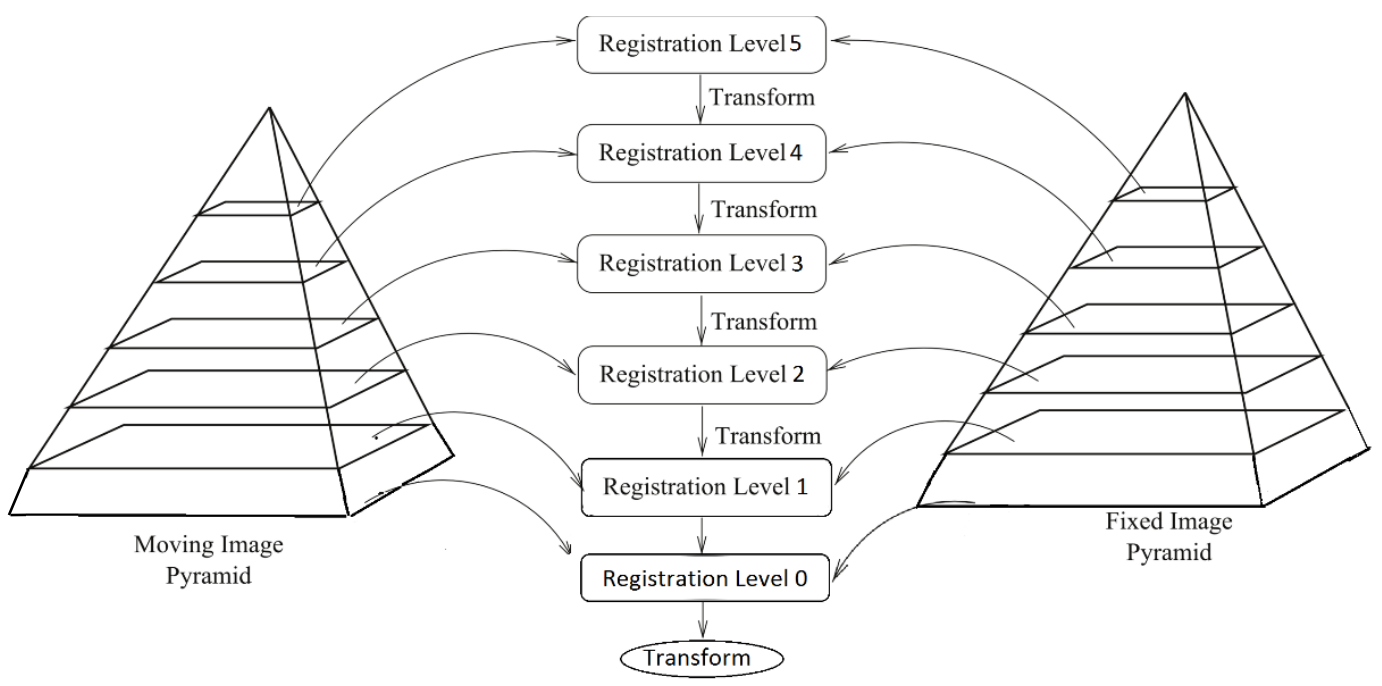

Figure 7: Pyramidal process used in YaDICs

DVC was performed between the reference image at minimum load and the deformed image at maximum load of each considered cycle. Displacement field measurements were performed for a region of interest of $1200 \times 1200 \times 601$ voxels ${ }^{3}$. Before displacement field measurements, all the tomographic images were first rigidly registered with the reference image, 
i.e., the image at the minimum load of the first cycle. Therefore, a calculation with several sequences of OFI searching all the translations and rotations between two images was computed before DVC measurement.

\subsection{INITIAL OPTIMIZATION OF DVC-ROUTINE}

General statistical methods for experimental data processing were computed to show the efficiency of the regularization with a median filter. A cube of $250 \times 250 \times 250$ voxels was chosen in a zone with a high strain concentration, and a comparison of standard deviation and mean values measured with different element sizes for DVC was realized. The results showed that the standard deviation of the strain is quite similar with an element size of $8 \times 8 \times 8$ voxels $(\operatorname{std}(\varepsilon)=0.0056)$ and with an element size of $4 \times 4 \times 4$ voxels $(\operatorname{std}(\varepsilon)=0.0047)$. The calculations of the mean strain confirm the preservation of the strain amplitude between an element size of $8 \times 8 \times 8$ voxels and $4 \times 4 \times 4$ voxels, since the $\varepsilon_{(\text {mean) }}$ is equal to 0.0179 and 0.0178 for an element size of $8 \times 8 \times 8$ voxels and $4 \times 4 \times 4$ voxels, respectively. The originalities of this platform are this regularization technique, which is quite new in the correlation methods, and also the multi-resolution scheme where different strategies can be adopted for different scales.

The spatial resolution is set by the size of element selected for DVC computation. In this work different sizes of isotropic element were tested with 16, 8 and 4 voxels edge. The results of these calculations are shown in the Fig.8. A phenomenon of diffusion appears due to the size of element. Indeed, when the size increases artefacts become visible around the crack. This phenomenon is essentially caused by the discontinuity of the crack. In fact, the median filter used allows conserving the discontinuities and the strain amplitude. By using the above parameters of correlation, one DVC measurement takes about 90 minutes with a size of element of 16 voxels edge, 150 minutes with 8 voxels edge and 480 minutes with 4 voxels edge. Although, the duration of the calculation is longer than the others, an element with a 4 voxels edge appears to be the best for this study, as the strain fields are localized in the same area than with larger elements but show less diffusion. With a1200x1200x601 voxels tomographic image and a 4 voxel element size, the number of Degrees of Freedom reaches 40 million.
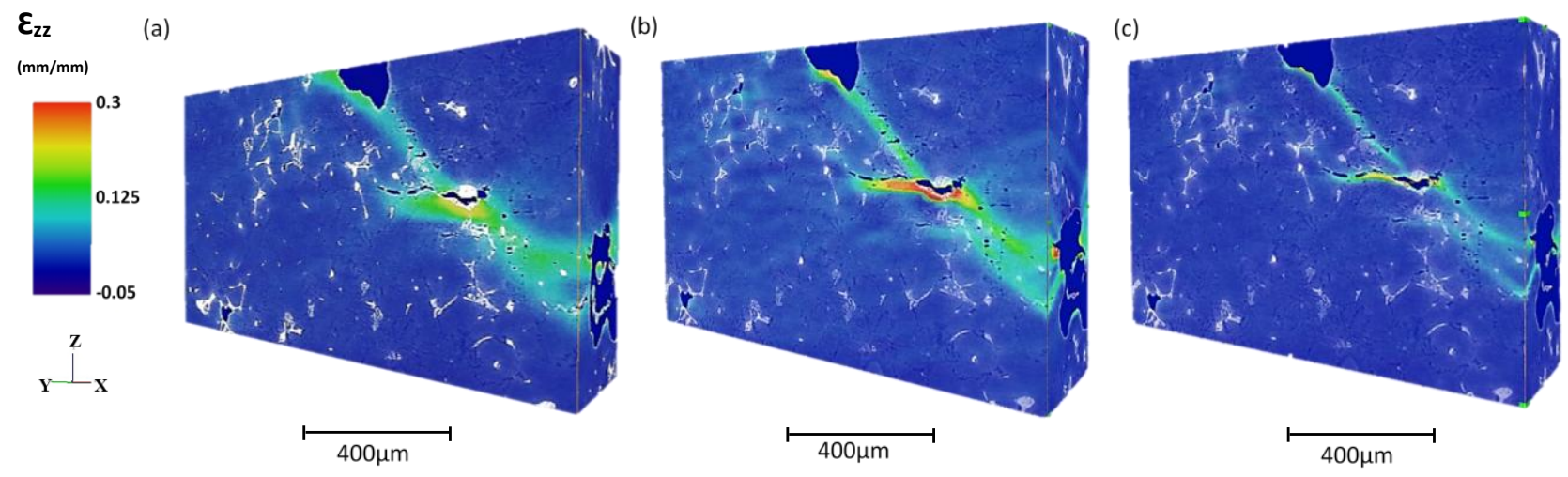

Figure 7: Strain field Ezz showing the effect of the element size on the localization of strain: (a) 16 voxels, (b) 8 voxels, (c) 4 voxels The uncertainty of the measured displacement field was calculated to assess the feasibility of DVC. This uncertainty is determined from the standard deviation of the displacement computed between two images, one at the reference position and another after a $10 \mu \mathrm{m}$ translation in a direction at $90^{\circ}$ with respect to the X-ray beam and to the rotation axis of the 
sample. Then, the standard deviation of the displacement is calculated for each component in the three different directions permitting to verify if there is one favoured direction in the noise. Figure 9 shows an uncertainty range from about 0.021 voxel $(0.058 \mu \mathrm{m})$ to 0.058 voxel $(0.16 \mu \mathrm{m})$ for element sizes ranging from 32 to 4 voxels $(88-11 \mu \mathrm{m})$. The spatial resolution of the measured displacement in 3D shows encouraging results. Although, the uncertainty for high spatial resolution is about three times higher than at low resolution, the strain localization around the crack at an element size of 4 voxel is better assessed than at coarser resolution. In what follows, the results are shown with a 4 voxel element size. Figure 10 represents the different scales used in this work: the sample analysed is about $2 \times 2 \mathrm{~mm}^{2}$ after mechanical polishing, the size of the pores from which the cracks are initiated varies between 450 and $1100 \mu \mathrm{m}$, and the smaller element used is $11 \mu \mathrm{m}$.

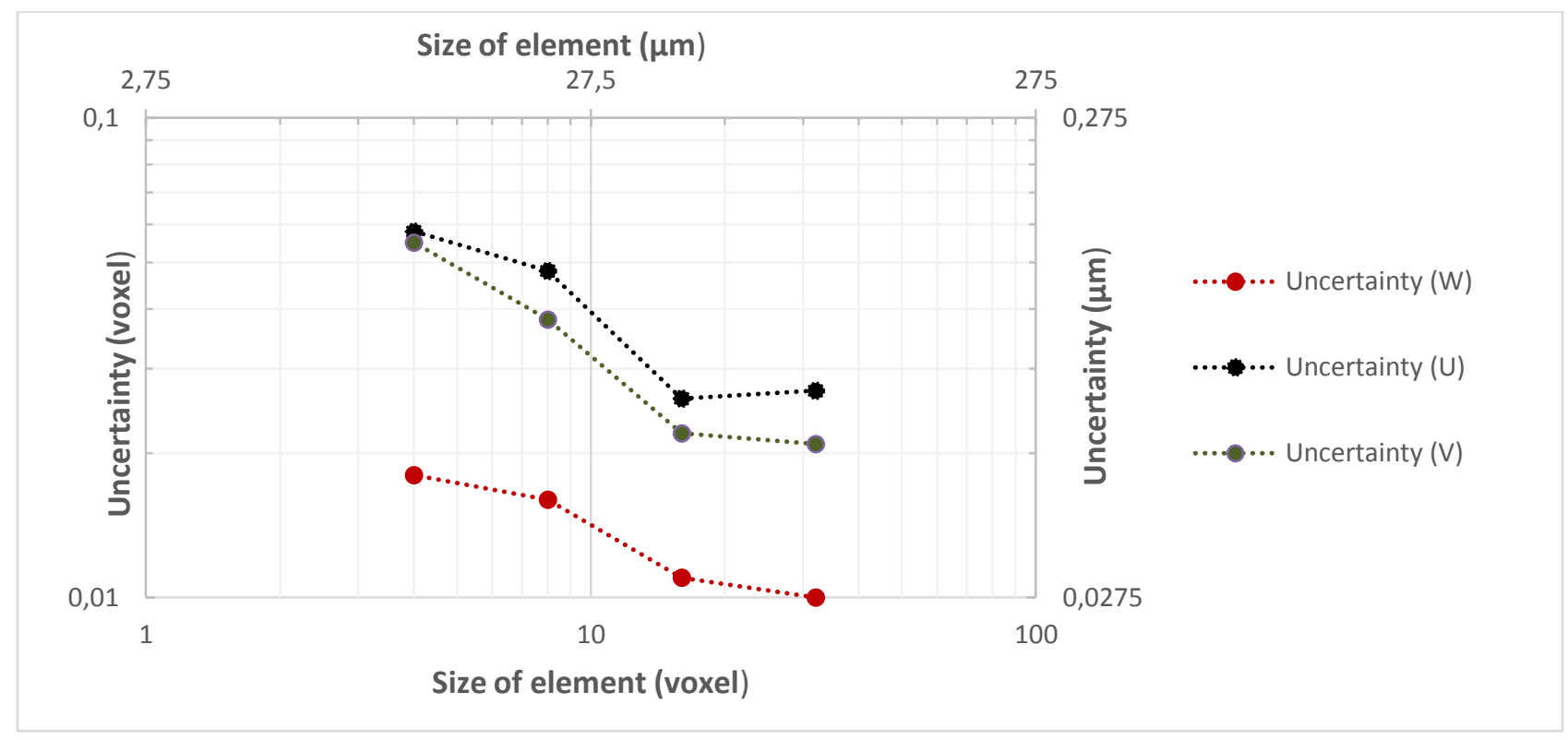

Figure 9: Uncertainty in displacement fields in the 3 directions measured with DVC

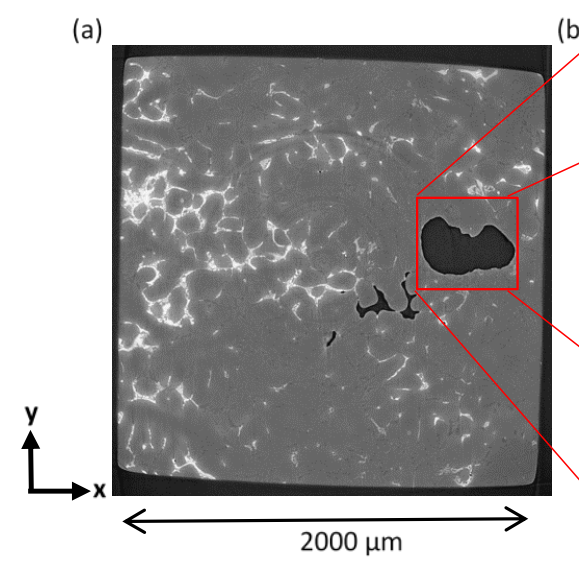

(b)
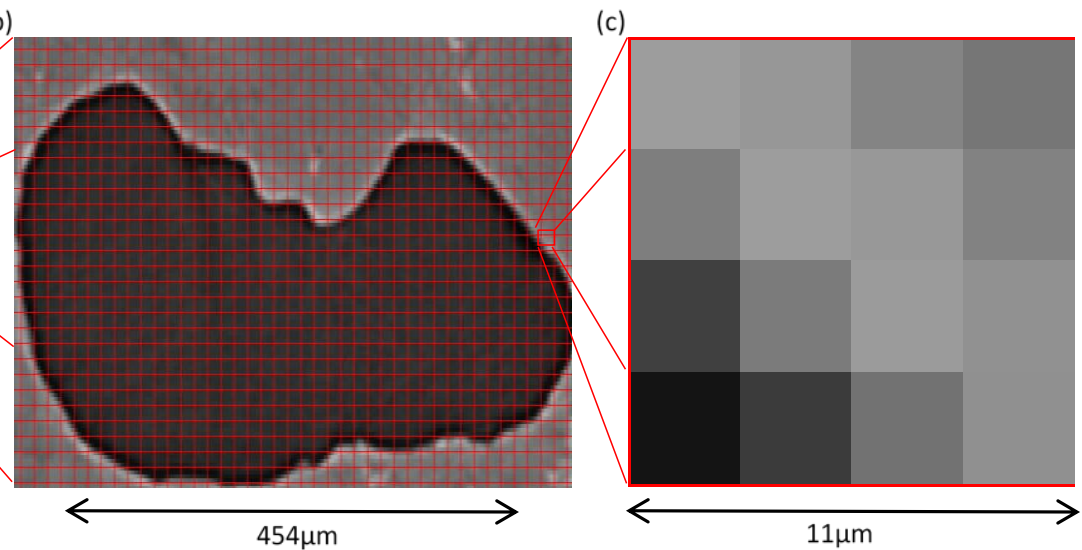

Figure 10: Schematic representation showing: (a) a reconstructed slice of the specimen ( $2 \times 2 \mathrm{~mm} 2 \mathrm{cross}$ section), (b) an enlarged view of the pore on which the grid of element used for DVC has been superimposed, (c) the voxel scale with such an element at the border between the matrix and the pore. 


\section{RESULTS AND DISCUSSION}

\subsection{Crack initiation}

For fatigue tests, a maximum stress of $75 \mathrm{MPa}$ was applied at $250^{\circ} \mathrm{C}$, with an expected average strain $\varepsilon_{z z}$ of $0.4 \%$ obtained from FE computation which is in accordance with an average strain of $0.5 \%$ measured by DVC between the minimum and the maximum loads of the first cycle. The in-situ tests highlighted the porosity as the main influent parameter on crack initiation as crack always initiated close to a pore, and often in the bulk independently of the temperature (Fig.11a\& b). Results obtained at room temperature [29] [30] at different numbers of fatigue cycles showed crack initiation close to the largest pores and crack propagation along the hard inclusions towards the free surface. At temperatures above $200^{\circ} \mathrm{C}$ (Fig.12), cracks were detected in silicon particles (Fig.12b) around the main pore that drove to failure but also in other areas of the specimen gauge length (Fig.12c). For all the specimens cracks initiated around large internal pores most of the time, during the first loading step.

(a)

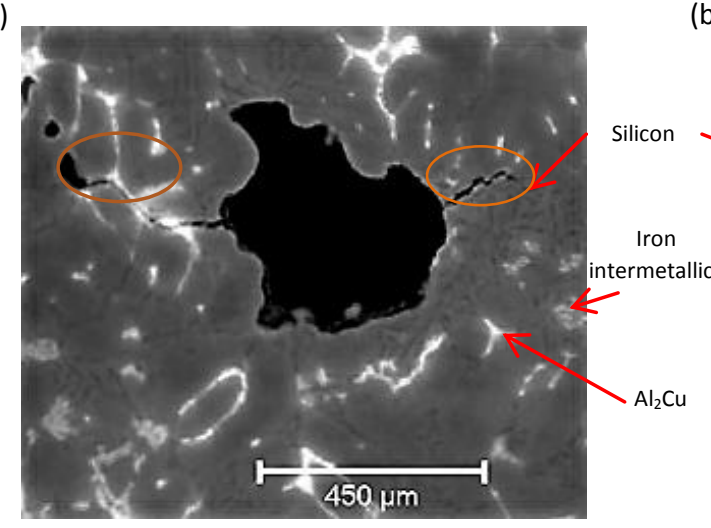

(b)

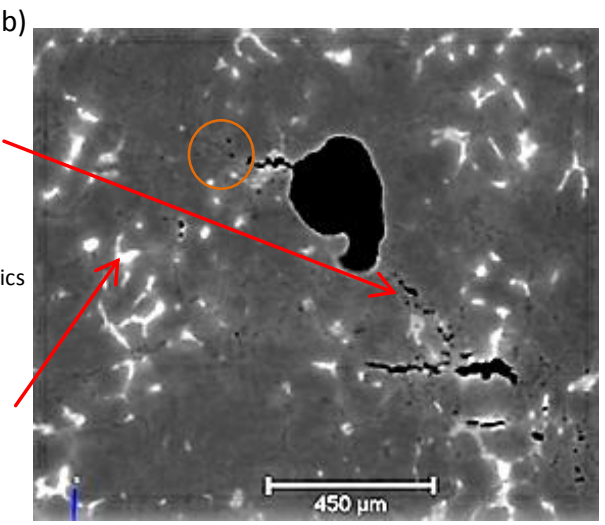

Figure 8: Tomography images showing cracks initiated from an internal pore at (a) $150^{\circ} \mathrm{C}(\mathrm{Ncycles}=10000$, ?max $=90 \mathrm{MPa})$, (b) $250^{\circ} \mathrm{C}($ Ncycles $=30$, ? $\max =75 \mathrm{MPa})$ 


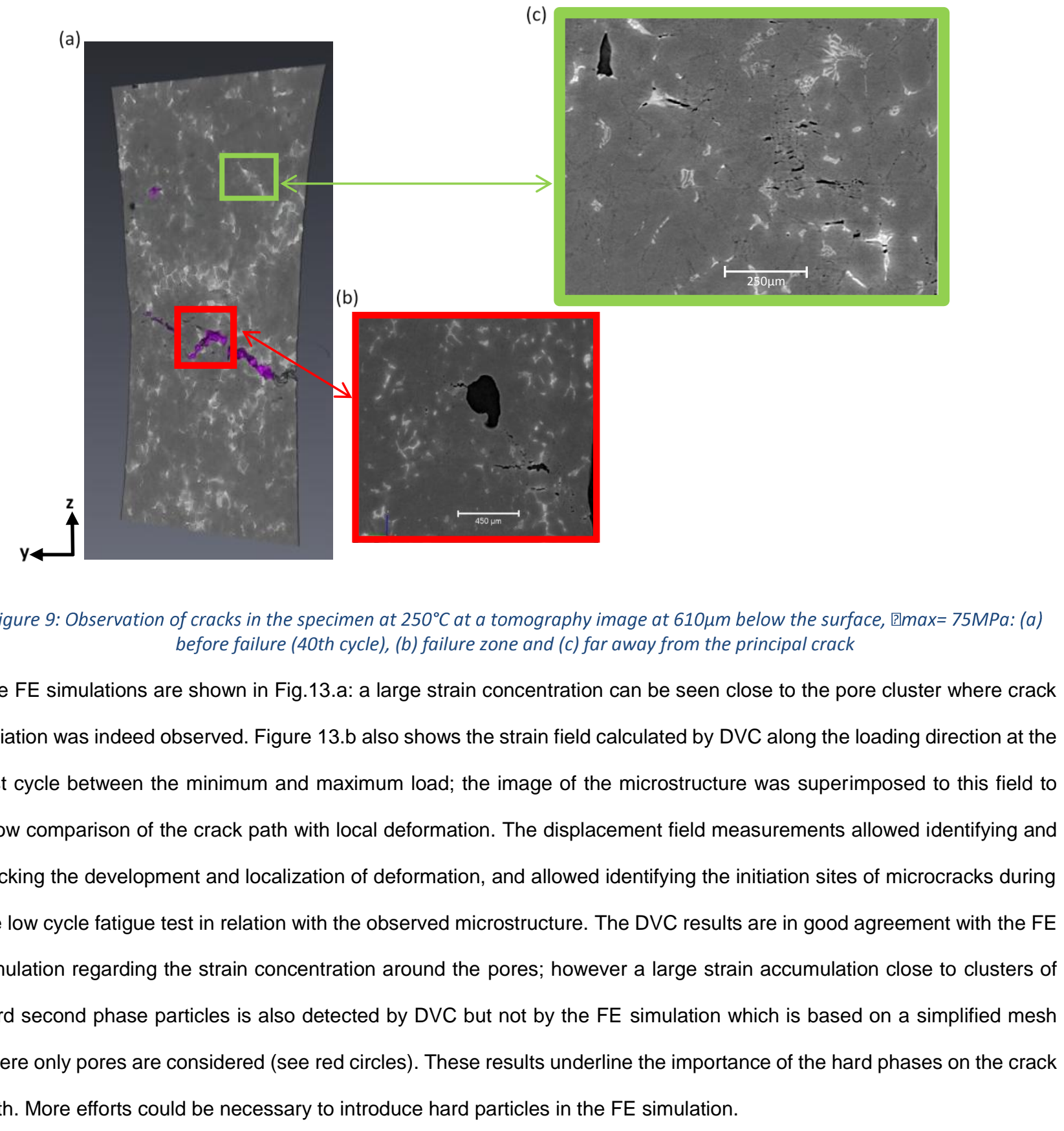




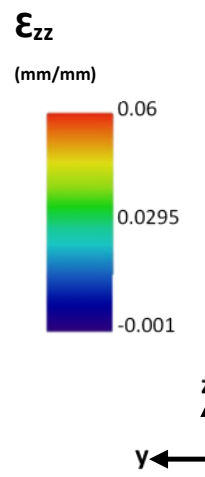

(a)

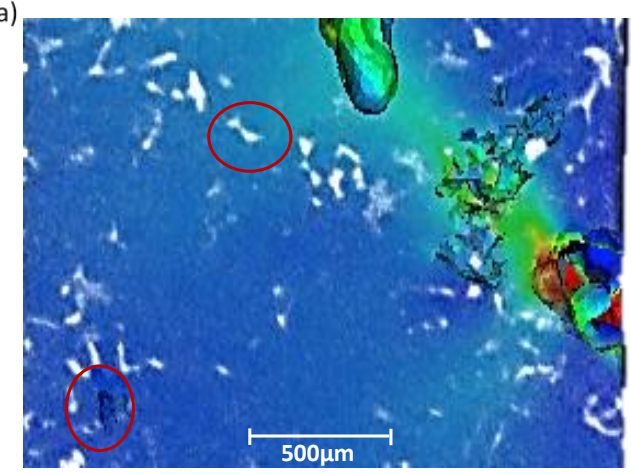

(b)

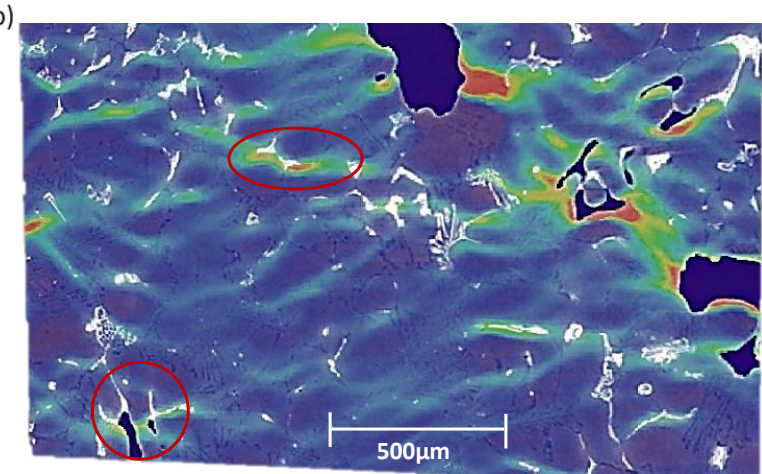

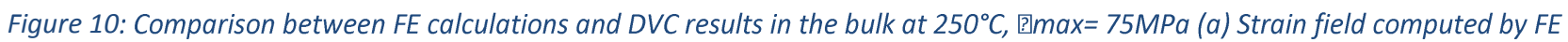
simulation during monotonic loading (elasto plastic calculation), i.e. at the maximum load of the first half-cycle of fatigue and (b) Strain field measured with DVC between the minimum and maximum of the first cycle; strain localization at hard second phase particles in the DVC results are circled in red.

\subsection{CRACK PROPAGATION}

A correlation between the localization of damage and the strain localization can be performed based on the reconstructed $3 \mathrm{D}$ images. The internal microstructure of the material as well as the displacement and strain fields at $250^{\circ} \mathrm{C}$ between the minimum and maximum of each cycle are shown on Fig.14 for different numbers of fatigue cycles. After the first cycle, a strain localization is noticed around a subsurface pore where the crack nucleated (see the arrow in Fig.14.c). After 10 cycles, the strain localization extends to be visible, especially in the area between the pore initiating the crack and a neighbouring pore (see the red circle in Fig.14.f). Crack propagation is observed in Fig.14.h\&i, after 30 cycles. The displacement discontinuities (Fig.14.b,e,h) visible in the crack zone are in good agreement with the strain localization (Fig.14.c,f,i) and the crack location (Fig.14.a,d,g). In this zone, cracks are observed in numerous phases. In order to understand the propagation, a zoom was made between the two pores. Figure 12 shows the strain localization at different cycles. After the first cycle (Fig.15.a), a strain localization is observable around an Al2Cu particle between the two pores, the local strain around the $\mathrm{Al}_{2} \mathrm{Cu}$ particle is about $\varepsilon_{\mathrm{zz}(\mathrm{Al} 2 \mathrm{Cu})}=2.6 \%$ while the mean strain is $\varepsilon_{\mathrm{zz} \text { (mean) }}=0.4 \%$. The strain localization is also observed after 5 cycles (Fig.15.b) on hard inclusions between the pores while no crack could be

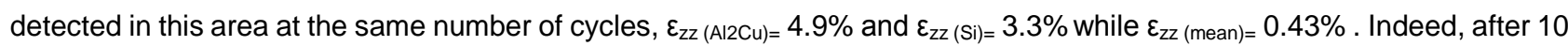
cycles (Fig.15.c), micro-cracks are observed in the $\mathrm{Al}_{2} \mathrm{Cu}$ particle and in the silicon particles, matching with the previous strain localization. After 20 cycles (Fig.15.d), the cracks propagate along the hard inclusions including iron intermetallics, copper containing phases and above all silicon phase leading to the final failure. From these observations, it appears that the presence of $\mathrm{Si}$ particles is an essential feature for the propagation of damage for the experimental conditions investigated here. Several explanations can be suggested to understand the role of Si particles on the propagation. The first one is that the silicon particles form an extended network [31] [32] in the whole volume of the specimen contrary to the copper and iron intermetallics phases [30]. The second explanation is the coarser morphology of the silicon particles which generate large stress concentration [33] in this material that has been Sr modified but has not undergone a solution heat treatment. Lastly, the eutectic silicon is more brittle than other hard inclusions at high temperatures [34]. Strain localizations were observed in zones where cracks propagations were observed. Moreover, the localization is noticeable before the 
crack observation and the strain level at cracked particles can now be quantitatively assessed thanks to DVC. The local strain level that leads to the cracking of particles ranges between 4 to $8 \%$ for the $\mathrm{Al}_{2} \mathrm{Cu}$ particles and between 3 to $6 \%$ for the Si particles.

This study highlights the possibility to understand the roles played by the pores and the various hard phases of the microstructure through the local measurement of strain. The DVC measurement is in accordance with the FE simulation based on a realistic 3D microstructure since the localization zones and the strain levels are quite equivalent. Some studies [35], [36] used FE computation to show the influence of pores and Si particles size, morphology and configuration on particle fracture in Al-Si alloy, but these analyses are realized for simplified microstructure that does not take in consideration the complex structure of the alloy. Therefore, the DVC technique gives more realistic results. Thanks to the high spatial resolution, a localization around the hard phases $\left(\mathrm{Al}_{2} \mathrm{Cu}\right.$ and $\left.\mathrm{Si}\right)$ in the neighbourhood of pores is pointed out. This level of representativeness could not be accessible by FE computation as it meets several difficulties such as the mesh size (expensive calculation) and also the mechanical properties of the hard phases which are poorly understood at $250^{\circ} \mathrm{C}$. Thus, the FE simulation allows only to have an idea of the crack location since it is around the pores that the crack nucleated. Then thanks to DVC measurement, the observation of the crack path through the cracked particles is possible. The 3D images allow access to the 3D arrangement of the hard phases to understand the complex crack path and extract the 3D crack. 
(a)

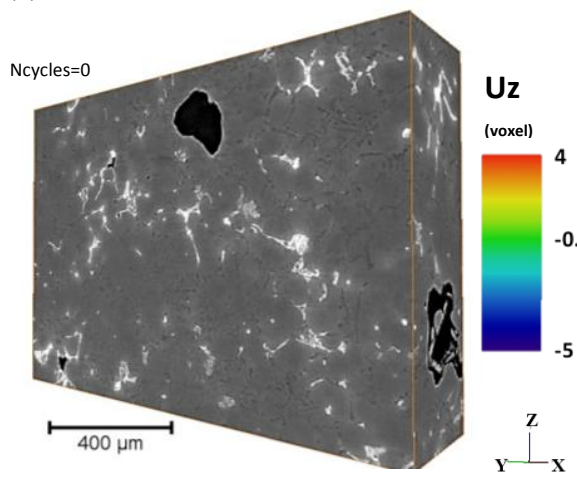

(d)

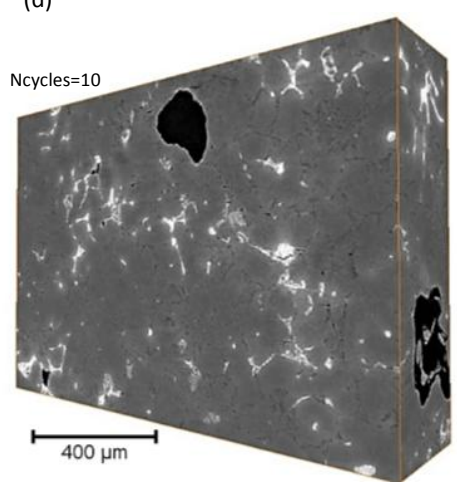

(g)
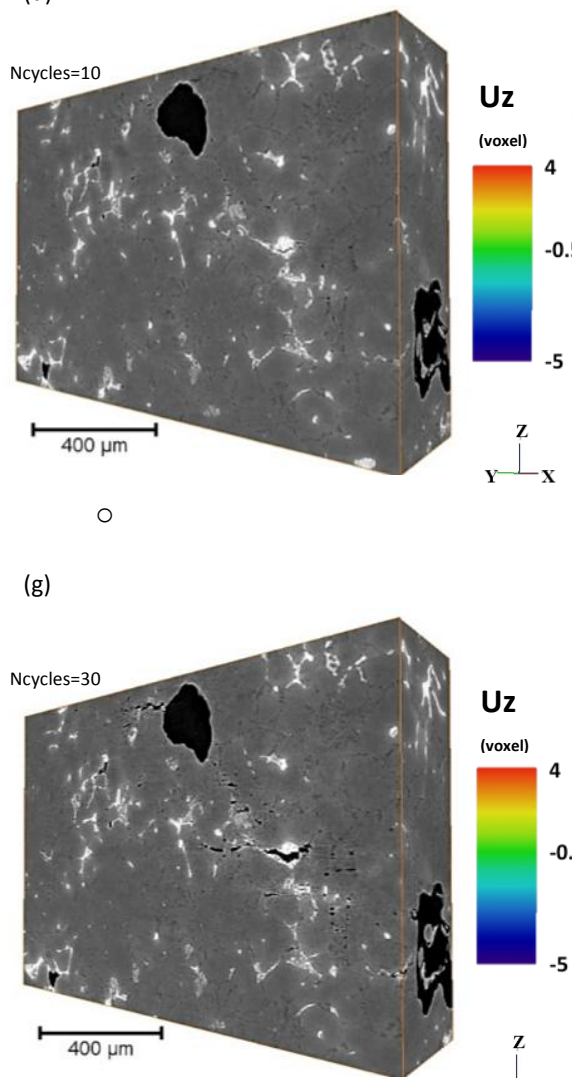

(b)

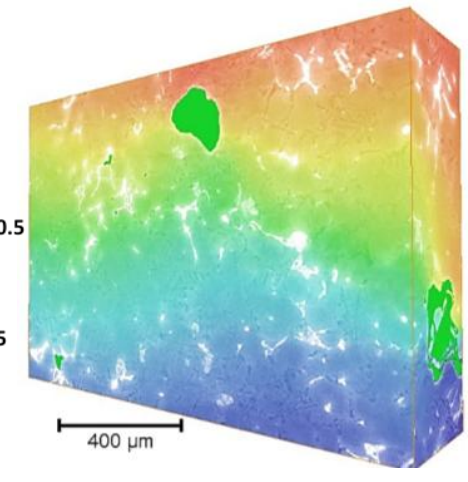

O

(e)

(h)

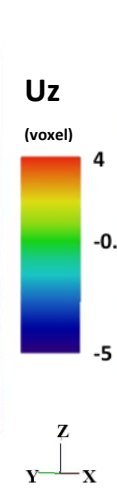

$\stackrel{L}{y}_{x}$

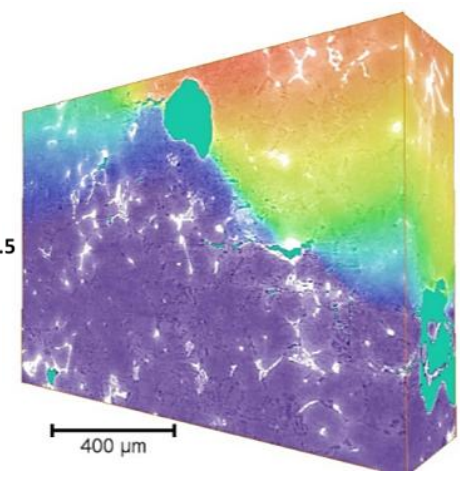

(c)

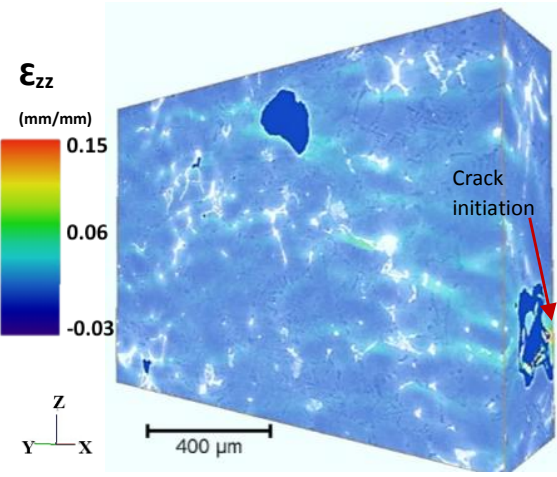

O

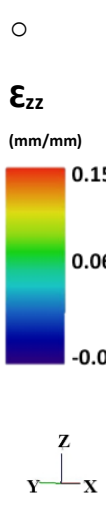

(f)

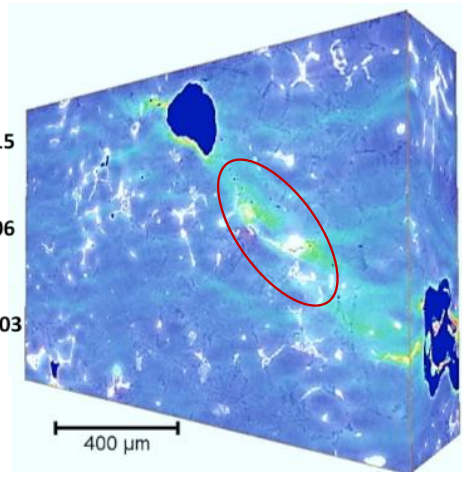

o

(i)

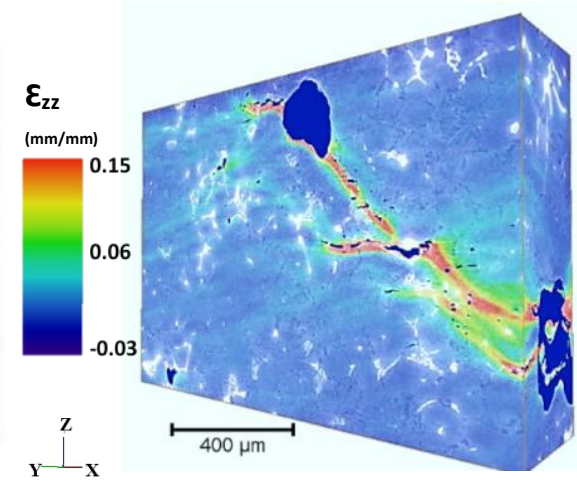

o

Figure 11: Slice view at $477 \mu \mathrm{m}$ below the surface, $\left(250^{\circ} \mathrm{C}\right.$, omax $\left.=75 \mathrm{MPa}\right)$, at $(a, b, c)$ Ncycles $=0,(d, e, f)$ Ncycles $=10$ \& $(g, h, i)$ Ncycles $=30$, showing $(a, d, g)$ the crack location, $(b, e, h) 3 D$ displacement field (in voxels; 1 voxel $=2.75 \mu m$ ) along the loading direction $z$ and $(c, f, i)$ ezz strain 

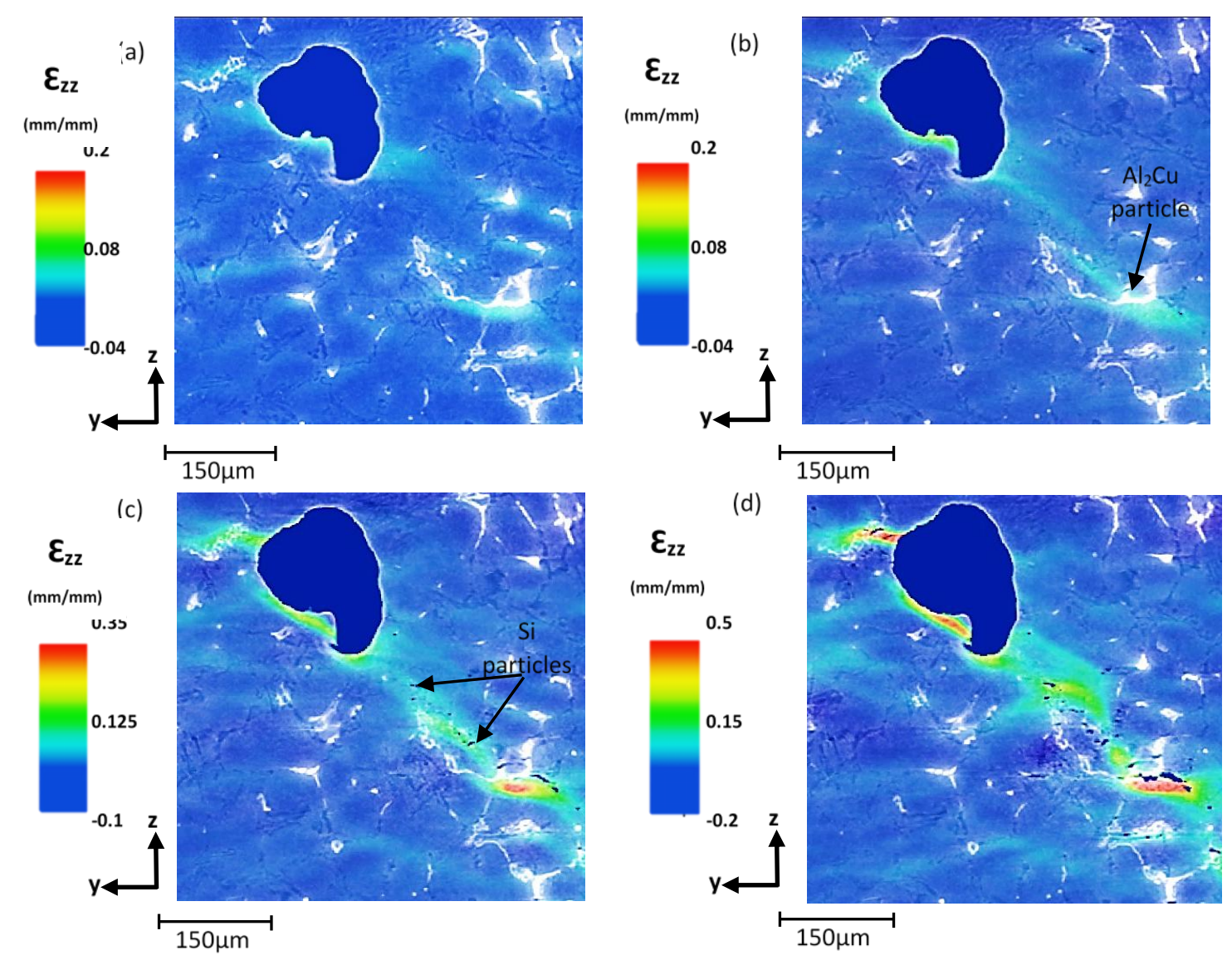

Figure 12: Zoom between the pores cluster showing strain localization $\varepsilon z z$ at: (a) Ncycle=0, (b) Ncycle=5, (c) Ncycle=10 and Ncycle $=20$

\section{Conclusions}

The efficiency of the experimental protocol using laboratory and synchrotron tomography, FE simulation and 3D DVC to study the influence of the casting microstructure upon the mechanical properties of an Al-Si alloy has been proved. To the best of the authors' knowledge, it is the first time that DVC images are obtained at high temperature in an optically opaque material and with such a resolution, i.e. at 4 voxel element size which results in a high Degree of Freedom (40 million). Thanks to the use of synchrotron X-rays, a detailed description of the material microstructure and, hence a high density of markers that can be used for the correlation can be obtained (detection of Si particles through phase contrast) and the scan duration (45s) is small enough to avoid any creep effect in the mechanically loaded sample with a voxel size in the micrometre range. DVC measurement allows to access local strain levels which help in understanding of the influence of the pores and hard phases on the fatigue mechanisms. Crack initiation is porosity driven while propagation is correlated with the presence of hard intermetallic phases. The crack nucleates during the first loading cycle and propagates along the hard phases until failure. Micro-cracks are mainly observed in the silicon particles due to the stress concentration around them although some cracks are also visible in the hard second phases such as $\mathrm{Al}_{2} \mathrm{Cu}$. These experiments at high temperature highlighted the role of pores on strain localization: the pores generate enough stress concentration to facilitate the propagation in this area. Hard phases and strain localization between pores seem to have a high influence in the propagation path. The feasibility of DVC measurement at this finer scale resolution was assessed by the uncertainty 
measured. DVC measurement with an isotropic element of 4 voxel edge is proved feasible with an uncertainty that remains at the sub voxel scale. This protocol also gives some very interesting information on the degree of complexity which has to be taken into account in so-called "realistic" microstructures to reproduce actual strain development within the samples.

\section{ACKNOWLEDGMENTS}

The authors wish to thank the ANR (Agence Nationale de la Recherche) MatetPro project INDiANA (ANR-12RMNP-0011) for funding the study on AI-Si aluminum alloys. The ESRF (European Synchrotron Radiation Facility) is also acknowledged for providing beamtime on the ID19 beamline; Elodie Boller and Vincent Fernandez from ESRF are particularly acknowledged for their help and advices with the Synchrotron tomography experiment. The ISIS4D X-Ray CT platform has been funded by International Campus on Safety and Intermodality in Transportation (CISIT), the Nord-Pas-de-Calais Region, the European Community and the National Center for Scientific Research. The authors gratefully acknowledge the support of these institutions.

\section{BIBLIOGRAPHY}

[1] S. Tabibian, E. Charkaluk, A. Constantinescu, F. Szmytka, and A. Oudin, "TMF criteria for Lost Foam Casting aluminum alloys: TMF OF LOST FOAM CASTING ALUMINUM ALLOYS," Fatigue Fract. Eng. Mater. Struct., vol. 36, no. 4, pp. 349-360, Apr. 2013.

[2] R. Albonetti, "Porosity and Intermetallic Formation in Lost Foam Casting of 356 Alloy," Univ. West. Ontario London, Ontario, 2000.

[3] Q. G. Wang, D. Apelian, and D. A. Lados, "Fatigue behavior of A356-T6 aluminum cast alloys. Part I. E€ ect of casting defects," vol. 1, pp. 73-84, 2001.

[4] X. Cao and J. Campbell, "The nucleation of Fe-rich phases on oxide films in Al-11.5 Si-0.4 Mg cast alloys," Metall. Mater. Trans. A, vol. 34, no. July, pp. 1409-1420, 2003.

[5] S. Tabibian, E. Charkaluk, A. Constantinescu, F. Szmytka, and A. Oudin, "TMF-LCF life assessment of a Lost Foam Casting A319 aluminum alloy," Int. J. Fatigue, vol. 53, pp. 75-81, Aug. 2013.

[6] J.-Y. Buffière, S. Savelli, P. H. Jouneau, E. Maire, and R. Fougères, "Experimental study of porosity and its relation to fatigue mechanisms of model Al-Si7-Mg0.3 cast Al alloys," Mater. Sci. Eng. A, vol. 316, no. 1-2, pp. 115-126, Nov. 2001.

[7] S. Tabibian, E. Charkaluk, A. Constantinescu, A. Oudin, and F. Szmytka, "Behavior, damage and fatigue life assessment of lost foam casting aluminum alloys under thermo-mechanical fatigue conditions," Procedia Eng., vol. 2, no. 1, pp. 1145-1154, Apr. 2010.

[8] S. Dezecot, J. Buf, A. Koster, V. Maurel, F. Szmytka, E. Charkaluk, N. Dahdah, A. El, N. Limodin, and J. Witz, "Scripta Materialia In situ 3D characterization of high temperature fatigue damage mechanisms in a cast aluminum alloy using synchrotron X-ray tomography," vol. 113, pp. 254-258, 2016.

[9] J.-F. W. S.Dezecot, J-Y Buffière, A.Koster, V.Maurel, F.Szmytka, E.Charkaluk, N.Dahdah, A.El Bartali, N.Limodin, "In-situ 3D characterization of high temperature fatigue damage mechanisms in a cast aluminium alloy using synchrotron X-ray tomography," Scr. Mater., no. Submitted for publication, 2015.

[10] P. Willmott, AN INTRODUCTION TO SYNCHROTRON RADIATION: TECHNIQUES AND APPLICATIONS. 2011.

[11] A. Kak and M. Slaney, "Principles of Computerized Tomographic Imaging," Engineering, p. 344, 1988.

[12] D. Paganin, S. C. Mayo, T. E. Gureyev, P. R. Miller, and S. W. Wilkins, "Simultaneous phase and amplitude extraction from a single defocused image of a homogeneous object," J. Microsc., vol. 206, no. 1, pp. 33-40, 2002.

[13] N. Limodin, J. Réthoré, J. Adrien, J. Y. Buffière, F. Hild, and S. Roux, "Analysis and Artifact Correction for Volume Correlation Measurements Using Tomographic Images from a Laboratory X-ray Source," Exp. Mech., vol. 51, no. 6, pp. 959-970, 2011.

[14] N. Limodin, J. Réthoré, J. Y. Buffière, F. Hild, S. Roux, W. Ludwig, J. Rannou, and A. Gravouil, "Influence of closure on the $3 \mathrm{D}$ propagation of fatigue cracks in a nodular cast iron investigated by $\mathrm{X}$-ray tomography and $3 \mathrm{D}$ volume correlation," Acta Mater., vol. 58, no. 8, pp. 2957-2967, 2010. 
[15] J. Rethore, N. Limodin, J.-Y. Buffiere, F. Hild, W. Ludwig, and S. Roux, "Digital volume correlation analyses of synchrotron tomographic images," J. Strain Anal. Eng. Des., vol. 46, no. 7, pp. 683-695, 2011.

[16] W. J. Coudert Sébastien, Seghir Rian, "http://yadics.univ-lille1.fr/wordpress/." .

[17] B. K. Bay, T. S. Smith, D. P. Fyhrie, and M. Saad, "Digital volume correlation: Three-dimensional strain mapping using X-ray tomography," Exp. Mech., vol. 39, no. 3, pp. 217-226, 1999.

[18] M. a Sutton, J. J. Orteu, and H. W. Schreier, "Image correlation for shape, motion and deformation measurements," Springer, New York, doi, vol. 49, no. 0, p. 6221, 2009.

[19] N. Limodin, J. Réthoré, J.-Y. Buffière, A. Gravouil, F. Hild, and S. Roux, "Crack closure and stress intensity factor measurements in nodular graphite cast iron using three-dimensional correlation of laboratory X-ray microtomography images," Acta Mater., vol. 57, no. 14, pp. 4090-4101, Aug. 2009.

[20] B. Pan, D. Wu, and Z. Wang, "Internal displacement and strain measurement using digital volume correlation: a least-squares framework,” Meas. Sci. Technol., vol. 23, no. 4, p. 045002, 2012.

[21] F. Hild and S. Roux, "Digital Image Correlation : From Displacement Measurement to identification of Elastic Properties- a Review," Strain, vol. 42, 69-80, no. February, 2006.

[22] J. Lachambre, J. Réthoré, A. Weck, and J.-Y. Buffiere, "Extraction of stress intensity factors for 3D small fatigue cracks using digital volume correlation and X-ray tomography," Int. J. Fatigue, vol. 71, pp. 3-10, Feb. 2015.

[23] A.-L. Cauchy, "Méthode générale pour la résolution des systèmes d'équations simultanées [Translated: (2010)]," Compte Rendu des S'eances L'Acad'emie des Sci., vol. 25, no. 2, pp. 536-538, 1847.

[24] D. Bertsekas, "Nonlinear programming," Proceedings of the Second Berkeley Symposium on Mathematical Statistics and Probability, J. Neyman (Ed.), University of California Press, Berkeley, \{CA\}. pp. 481-492, 1993.

[25] P. Cachier, E. Bardinet, D. Dormont, X. Pennec, and N. Ayache, "Iconic feature based nonrigid registration: the PASHA algorithm," Comput. Vis. Image Underst., vol. 89, no. 2-3, pp. 272-298, 2003.

[26] J. K. Mandal and S. Mukhopadhyay, "Image Filtering Using All Neighbor Directional Weighted Pixels : Optimization Using Particle Swarm Optimization,” vol. 2, no. 4, pp. 187-200, 2011.

[27] G. Qiu, "An improved recursive median filtering scheme for image processing," IEEE Trans. Image Process., vol. 5, no. 4, pp. 646-648, 1996.

[28] E. Arias-Castro and D. L. Donoho, "Does median filtering truly preserve edges better than linear filtering?," Ann. Stat., vol. 37, no. 3, pp. 1172-1206, 2009.

[29] E. C. and J. Y. B. N.Dahdah, N.Limodin, A.El Bartali , J.F.Witz, R.Seguir, "STUDY OF DAMAGE MECHANISMS IN A319 ALUMINIUM ALLOY BY X-RAY TOMOGRAPHY AND DIGITAL VOLUME CORRELATION," in 3DMS, 2014.

[30] L.Wang, "Influence of the casting microstructure on damage mechanisms in Al-Si alloys by using 2D and 3D in-situ analysis," 2015.

[31] Z. Asghar, G. Requena, and E. Boller, "Three-dimensional rigid multiphase networks providing high-temperature strength to cast AlSi10Cu5Ni1-2 piston alloys.," Acta Mater., vol. 59, no. 16, pp. 6420-6432, 2011.

[32] F. Lasagni, a. Lasagni, M. Engstler, H. P. Degischer, and F. Mücklich, "Nano-characterization of Cast Structures by FIB-Tomography," Adv. Eng. Mater., vol. 10, no. 1-2, pp. 62-66, 2008.

[33] F. Barrirero, J., Engstler, M., and Mücklich, "Atom Probe Analysis of Sr Distribution in AlSi Foundry Alloys.," in In Light Metals 2013, B.A. Sadler, ed. (John Wiley \& Sons, Inc.), pp. 289-296., 2013.

[34] C. L. Chen, G. D. West, and R. C. Thomson, "Characterisation of Intermetallic Phases in Multicomponent Al-Si Casting Alloys for Engineering Applications," 2006.

[35] K. Gall, M. Horstemeyer, D. L. Mcdowell, and J. Fan, "Finite element analysis of the stress distributions near damaged Si particle clusters in cast AltSi alloys," vol. 32, pp. 277-301, 2000.

[36] J. Fan, D. L. McDowell, M. F. Horstemeyer, and K. Gall, "Cyclic plasticity at pores and inclusions in cast Al-Si alloys," Eng. Fract. Mech., vol. 70, no. 10, pp. 1281-1302, Jul. 2003. 